\title{
Tumour differentiation
}

\section{Differentiation in breast cancer}

\author{
STRETTON YOUNG, LILLIAN S. C. PANG, AND IAIN GOLDSMITH \\ From the Department of Pathology, Imperial Cancer Research Fund, Lincoln's Inn Fields, London
}

The Shorter Oxford English Dictionary defines differentiation in its biological sense as 'the process or the result of the process by which in the course of development a part, organ, etc, is modified into a special form, or for a special function; specialization'. Fully differentiated tissue is normal tissue with normal structure and function, and every tumour is less well differentiated than its parent tissue in one or more respects. Characteristics lost may be divided roughly into two classes, morphological and functional, and the degree of differentiation will be in inverse proportion to the extent to which these characteristics have been lost.

Since histopathologists practise a visual discipline, they tend to think of tumour differentiation in the morphological sense and the subject is important to them in attempting to assess the behaviour of a tumour and therefore the patient's prognosis. It is generally believed that structurally well differentiated tumours grow more slowly, metastasize less readily, and take longer to kill their hosts than their more anaplastic counterparts, and methods of grading tumours according to their degree of structural differentiation have been used for many years.

A good example of correlation between structural differentiation and growth rate is given by experiments of Knox, Linder, and Friedell (1970). Nine rat mammary tumours that had arisen spontaneously or been induced by chemicals or irradiation were used. The tumours were maintained by serial transplantation and were known to be stable in structure from one transplant generation to the next. Histology of these tumours was submitted to eight pathologists who arranged them in order of relative differentiation. The growth rate of transplanted pieces of tumour was found to correlate inversely and well with the degree of differentiation $(r=0 \cdot 77)$.

In human practice several different systems for the histological grading of breast cancer have been devised and opinions are divided as to their value. In 1950 Bloom published details of a comparatively simple system in which histological grading based on subjective estimates of three parameters (tubule formation, nuclear pleomorphism, and hyperchromatism and mitosis) appeared to be of prognostic value. The correlation between forecast and actual courses of the disease was even better if histological grade was combined with clinical stage. Later, Bloom, Richardson, and Harries (1962) compared histological grade with survival time in 86 cases of untreated breast cancer. The mean survival time was longest in cases with the best histological differentiation and shortest in those with the most anaplastic tumours. Clearly the less well differentiated tumours were more aggressive.

Many tumours show some evidence of functional differentiation. Often this is interesting but relatively unimportant, as in the case of carcinomas that keratinize or secrete mucin. Sometimes, however, the secretion of a functionally active tumour produces systemic effects, or the growth of the tumour can be influenced remotely by hormonal means.

Systemic effects can be produced by secretion of hormones from tumours of the endocrine glands such as the ovaries, adrenals, and pituitary. These hormonal effects are due to continued activity by the tumour of a function that is normally possessed by its parent epithelium. They are thus examples of functional differentiation. Remote effects can also be produced by some tumours by the secretion of polypeptide hormones that are not normally secreted (in physiological amounts at any rate) by the organs from which the tumours are derived. Included among such tumours are oat cell carcinomas of bronchus, carcinoid tumours of gut, medullary carcinoma of thyroid (Ross, 1972). Since these hormones are not normally associated with the tissue of origin it seems reasonable not to consider them as examples of functional differentiation and to refer to them instead as ectopic or inappropriate.

Another type of evidence of functional differentiation is found in tumours derived from hormonesensitive epithelia, and cancers of such epithelia, eg, breast, prostate, and uterus, can manifest a function of their parent tissue and undergo regres- 
sion after appropriate hormonal treatment. Clearly the tumours that respond in this way are those that bear a close functional resemblance to their parent tissue.

Remissions of breast cancer were induced by oophorectomy more than 70 years ago (Beatson, 1896) and by adrenalectomy 20 years ago (Huggins and Bergenstal, 1952). Hormonal treatment of one sort or another has come to be widely used and although remission rates of 30,40 , and $50 \%$ are reported, it is not yet possible to predict which cases will respond favourably and which will not. This is obvious from the reported remission rates, for if prediction were possible, remission in selected cases would be $100 \%$. The reason for this inability to predict remissions after hormonal treatment seems to be the inability to determine which tumours are composed of cells whose proliferation is hormonedependent.

In the normal mammary gland secretion and cell proliferation are each under hormonal control. It seems possible therefore that cells that are functionally differentiated in one of these respects might also be functionally differentiated in the other. Hormonally controlled secretory ability might, therefore, be an indication of hormonally controlled proliferation.

It has recently been shown that milk-specific proteins (principally casein) can be detected in paraffin sections of lactating mammary glands of rats (Young and Nelstrop, 1970). The present paper describes some preliminary results after the application of this method to sections of human breast cancer.

\section{Materials and Methods}

The materials and methods are similar but not identical to those described by Young and Nelstrop.

Pooled human milk was obtained from the maternity departments of St Thomas's and the Central Middlesex Hospitals. It was refrigerated as soon as possible and was stored at $-20^{\circ} \mathrm{C}$.

SEPARATION AND PURIFICATION OF MILK PROTEINS

About $100 \mathrm{ml}$ of the frozen milk was warmed to $37^{\circ} \mathrm{C}$. It was subjected to centrifugation at $1000 \mathrm{~g}$ for 20 minutes and the fat layer was removed. Further centrifugation of the defatted milk at 30$35000 \mathrm{~g}$ for 30 minutes caused the casein to be deposited. The deposited casein was washed several times with distilled water and resuspended in a small amount of distilled water. The yield of casein from human milk was considerably lower than from rat milk.

$4^{*}$
PREPARATION OF ANTIBODIES

Rabbits were immunized against human casein. They were given $0.5 \mathrm{mg}$ of casein in Freund's complete adjuvant (FCA) subcutaneously, followed by three booster doses of $1 \mathrm{mg}$ casein in saline intramuscularly.

Globulins were precipitated from the serum by $40 \%$ saturated ammonium sulphate, washed in $40 \%$ saturated ammonium sulphate, taken up in saline, and reprecipitated twice again. Some of the globulins were conjugated to fluorescein isothiocyanate(FITC) but most were left unconjugated for use with the indirect fluorescent antibody method. Both conjugated and unconjugated globulins were absorbed with human liver powder, human kidney powder, and with whole human serum whose proteins had been insolubilized but not denatured by treatment with ethyl chloroformate (Avrameas and Ternynck, 1967). Debris was removed from the globulins by filtering through a Swinny Millipore filter. Antibodies to rabbit immunoglobulins were obtained commercially and were already conjugated.

\section{HISTOLOGICAL METHODS}

Paraffin sections of formaldehyde-fixed human breast cancer were received from the Departments of Pathology of Guy's and New Cross Hospitals. The patients had been treated at the Breast Unit, Guy's Hospital. Urinary androgen and 17-hydroxycorticosteroid metabolites have been measured and the clinical history is available for each case. The sections were dewaxed, taken to water and soaked in $0.9 \%$ phosphate-buffered saline at $\mathrm{pH} 8$ for five minutes. Unconjugated antibody was applied for 15 minutes at $37^{\circ} \mathrm{C}$ and the sections were rinsed in saline at $\mathrm{pH} 8$ for five minutes, stained with conjugated antigammaglobulin for 15 minutes, rinsed twice at pH8 for five minutes each time, and mounted in glycerol/PBS buffered to pH8.

Paraffin sections of known positive material were also stained. These included normal lactating human mammary gland and portions of synthetic sponge (Spontex) that had been soaked in human milk, fixed, processed, embedded, and cut in the usual way. Control sections appropriate to the indirect methods were also stained. After examination and photography, some of the sections were overstained with haematoxylin and eosin and the same fields were rephotographed.

All the haematoxylin- and eosin-stained sections are housed in the Tumour Reference Collection (TRC) of the Imperial Cancer Research Fund and are referred to in the text and in the legends by their TRC numbers.

MICROSCOPY

All examinations and photographs were carried out 
on the same Zeiss WL microscope. The ultraviolet light source was an HBO 200 burner in an external housing with quartz collecting lens and quartz substage condenser. The lamp filter was UG 1 and barrier filters were 41 and -65 (Zeiss). Photography of fluorescent images was carried out on Kodak high speed Ektachrome $35 \mathrm{~mm}$ film developed to give an effective speed of 640 ASA.

GEL DIFFUSION

Immunodiffusion was carried out using $1.5 \%$ special agar-Noble in phosphate-buffered saline.

A 3 in $\times 2$ in. slide was covered with $4 \mathrm{ml}$ agar and the reactant wells were prepared with a Shandon well cutter.
The plate was developed for 48 hours at $4^{\circ} \mathrm{C}$, washed in phosphate-buffered saline, dried, and stained with $0 \cdot 1 \%$ amido black in $7 \%$ acetic acid.

\section{Results}

Two precipitation lines were found on the geldiffusion plates between anticasein that had been absorbed with liver, kidney, and serum on the one hand and total milk proteins on the other but only one line was seen between anticasein and casein. No line was detectable between the anticasein and whole serum (fig 1). A single line was also seen between casein and anticasein after the latter had been absorbed with human liver powder, with both liver
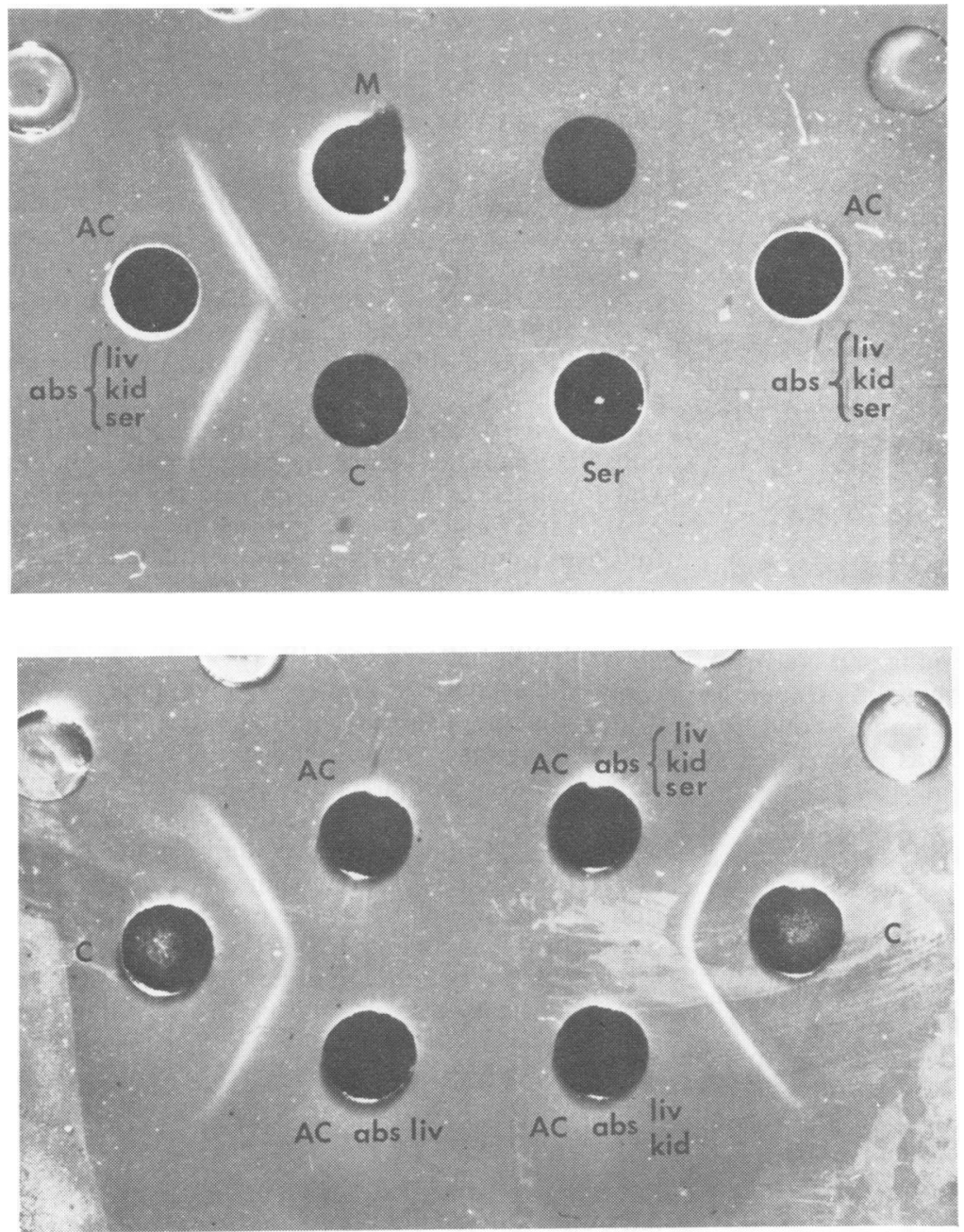

Fig 1 Gel-diffusion plate to show precipitation lines between anticasein that had been absorbed with liver, kidney, and serum ( $A C$ abs) on the one hand and milk $(M)$, casein $(C)$, and serum (Ser) on the other. $\times c 3$.

Fig 2 Gel-diffusion plate to show precipitation lines beween casein $(C)$ and anticasein $(A C)$ after different regimes of absorption of the latter. $\times c 3$. 
and kidney powders, and with liver, kidney, and serum (fig 2).

\section{POSITIVE CONTROL SECTIONS}

Paraffin sections of normal lactating human mammary gland were stained by the indirect method. Secretion, extracellularly located within the acini, fluoresced brightly and had a granular appearance (fig 3). Some intracellular fluorescence was also seen but was not widespread in the sections examined. Fluorescence was not seen after appropriate substitutions had been carried out in the method. Paraffin sections of synthetic sponge that had been soaked in human milk gave similar results to normal human mammary gland.

\section{BREAST CANCER}

All the 25 cases of breast cancer so far examined have contained evidence of some protein secretion in some part of the sections. Fluorescence has keen found in most of the tumour areas. Usually it has been extracellular and the secretion has formed a layer on the luminal surface of malignant acini or tubules. Intracellular fluorescence has been less common,

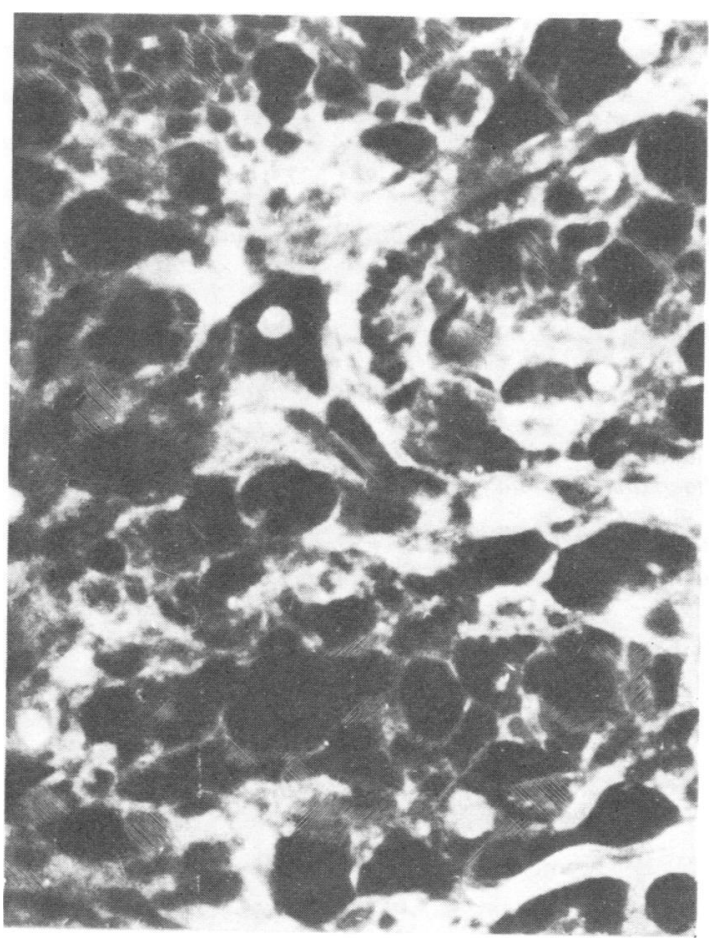

Fig 3 Lactating human mammary gland. Fluorescence mainly extracellular and intraacinar. Indirect fluorescent antibody method. $\times 720$. but has been seen in two forms: brightly fluorescent intracytoplasmic foci, and a diffuse fluorescence of the cytoplasm of individual tumour cells. Many of the sections of tumour have included areas of normal mammary gland and fluorescence has often been found on the luminal surface of normal ducts and somewhat less commonly within the acini.

The exact location and distribution of secretory fluorescence has varied from case to case and also from area to area within the same section; most cases have shown fluorescence in more than one location. The number of cases studied is too small to justify detailed numerical analysis but so far there has been no clear correlation between amount or nature of secretory fluorescence and degree of structural differentiation. Tumours have been examined in which almost complete lack of structural differentiation was accompanied by diffuse intracellular fluorescence of most of the neoplastic cells.

These points can be illustrated from three cases.

\section{Case 1: Intraluminal fluorescence (TRC 3674)}

This was a moderately well differentiated adenocarcinoma. The cells were small, moderately uniform in size and shape and with a moderate number of hyperchromatic cells and mitotic figures. Nucleoli were inconspicuous. Stroma was profuse in some areas with a good deal of hyalinization and some areas of elastosis (fig 4). The lumen of many of the tubule-like structures was lined by eosinophilic amorphous material and contained amorphous debris (fig 5). These tubule contents fluoresced brightly and comparatively uniformly throughout the tumour area (fig 6). In this case, therefore, moderately good structural differentiation was accompanied by widespread evidence of secretory activity.

Case 2: focal intracytoplasmic secretion (TRC 3675) This was a poorly differentiated carcinoma. The cells were, on the whole, small but varied a good deal in size and shape. Hyperchromatic cells and mitotic figures were moderately numerous and in some areas the cytoplasm was vacuolated. Stroma varied from area to area and there was very little evidence of tubule or acinus formation (figs 7 and 8). There was little evidence of secretory activity in this tumour but in a few areas there were brightly fluorescent foci of secretion located within the cytoplasm (fig 9). In this case poor structural differentiation was accompanied by only slight secretory activity.

Case 3: diffuse intracytoplasmic fluorescence (TRC 3679)

This was a poorly differentiated carcinoma. The 


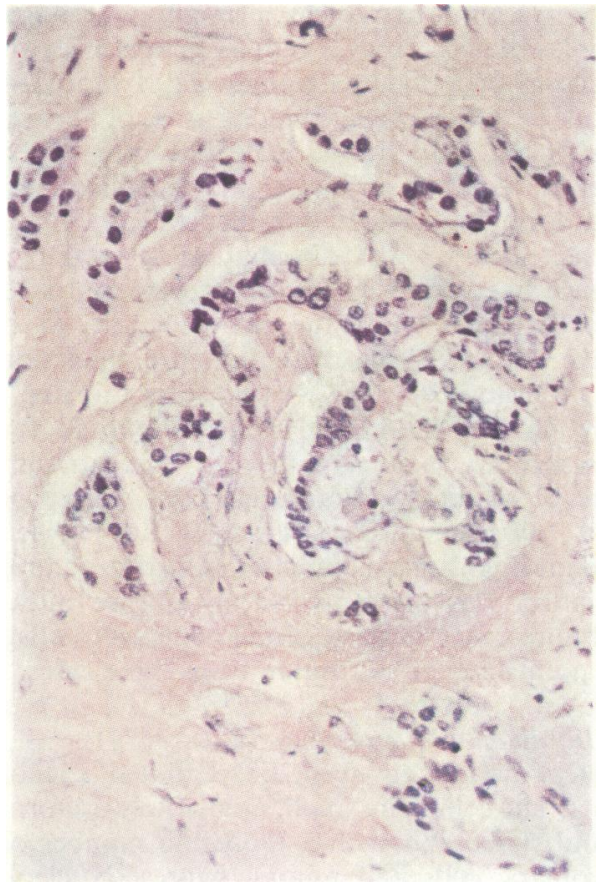

Fig 4

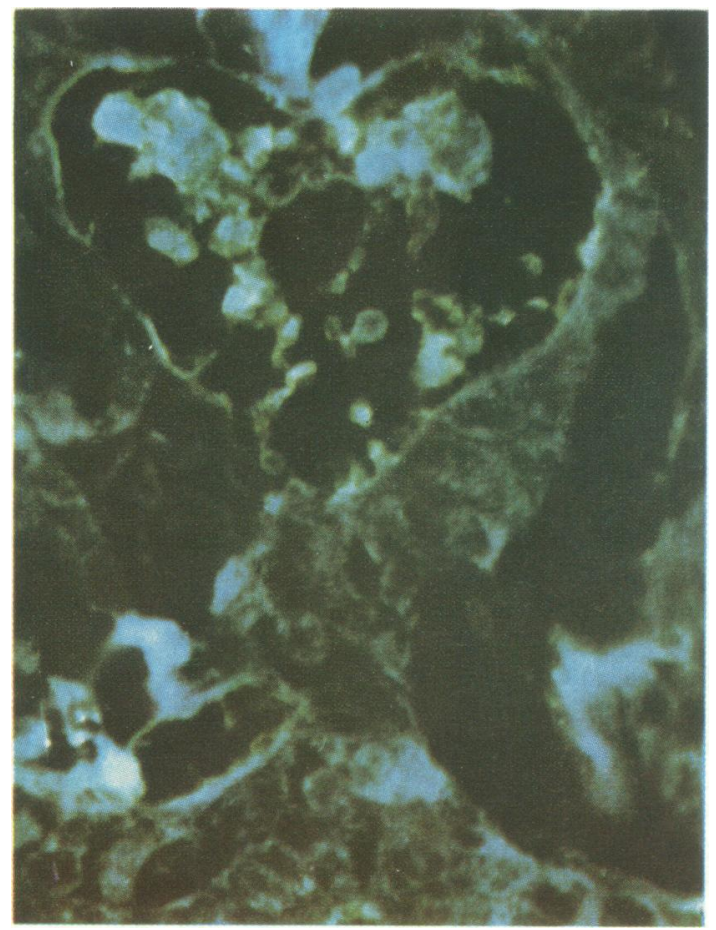

Fig 6

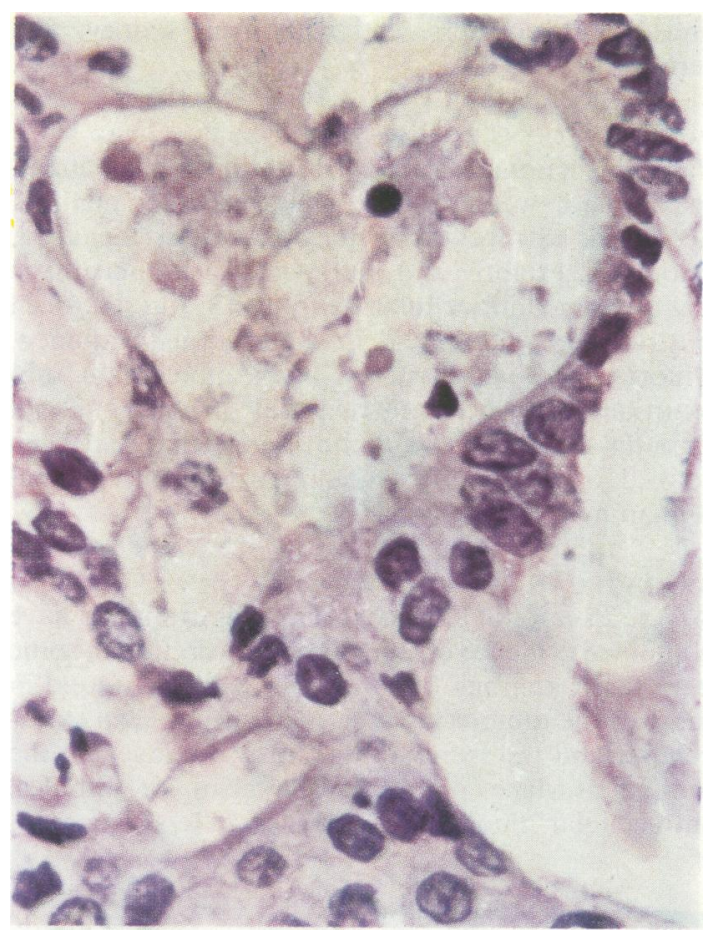

Fig 5

Fig 4 Moderately well differentiated adenocarcinoma (TRC 3674). Haematoxylin and eosin. $\times 200$.

Fig 5 Higher power view of part of fig 4 and same field as figure 6. Tubule-like structures lined by eosinophilic amorphous material containing eosinophilic debris. Section previously stained by indirect fuorescent antibody method now overstained with haematoxylin and eosin. $\times 720$.

Fig 6 Same field as fig 5 with original staining by indirect fluorescent antibody method. Secretory fluorescence on luminal border of neoplastic acinus and on luminal debris. $\times 720$. 


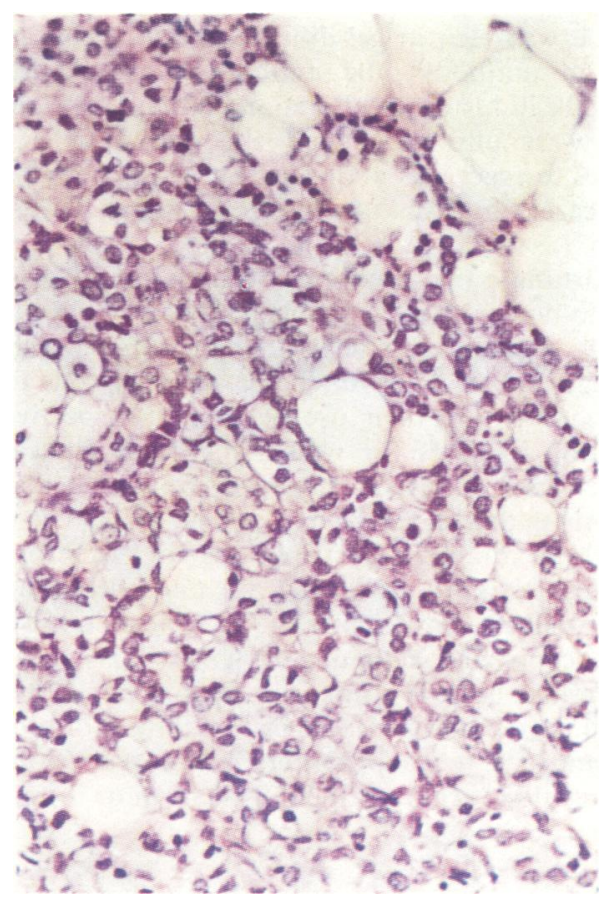

Fig 7

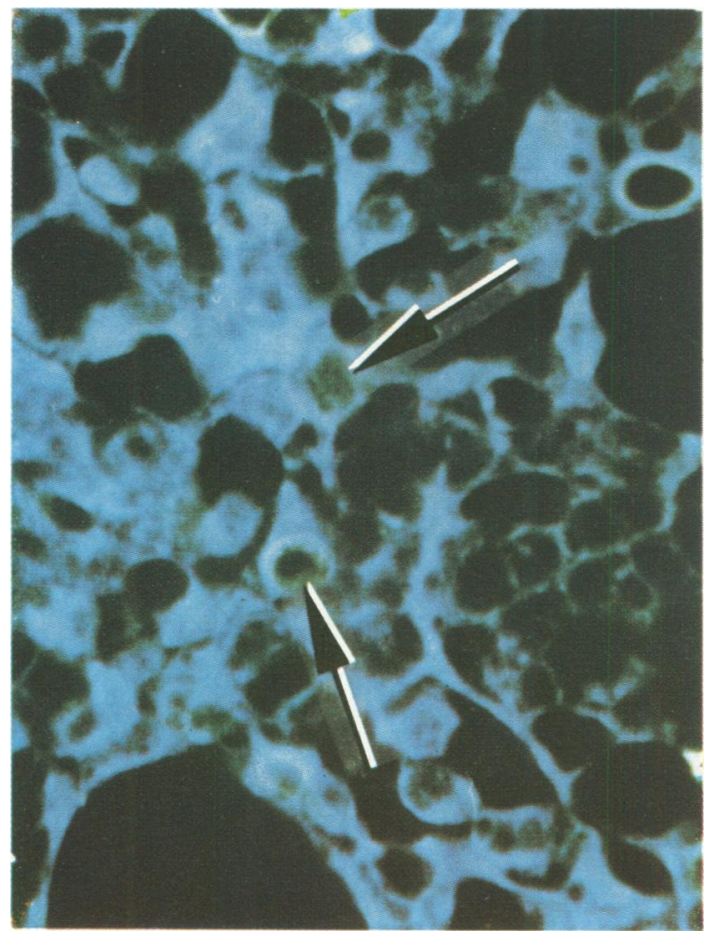

Fig 9

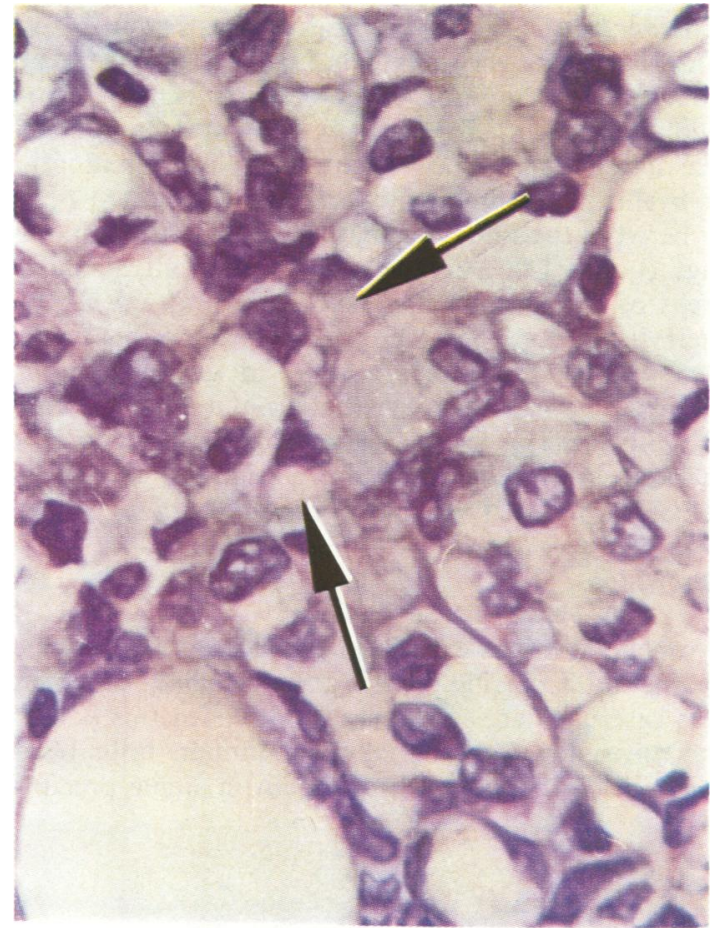

Fig 8

Fig 7 Poorly differentiated adenocarcinoma (TRC 3675). Haematoxylin and eosin. $\times 200$.

Fig 8 Higher power view of part of fig 7 and same field as figure 9. Intracellular vacuoles marked by arrows fluoresced brightly when previously stained by indirect fluorescent antibody method. Section now overstained with haematoxylin and eosin. $\times 720$.

Fig 9 Same field as fig 8 with original staining by indirect fluorescent antibody method. Fluorescent intracytoplasmic vacuoles marked with arrows. $\times 720$. 
cells were small, somewhat uniform in size and shape and with only a very small amount of cytoplasm that was often eosinophilic. Hyperchromatic nuclei, nucleoli, and mitotic figures were inconspicuous. Neoplastic cells formed no structures but lay individually or in short cords in the moderately abundant stroma or between the lipocytes that lay outside the tumour (figs 10 and 11). Many of the tumour cells in most areas of the tumour showed diffuse fluorescence of their cytoplasm (figs 12 and 13). Fluorescence was also seen within the lumina of a group of nonmalignant ducts that were adjacent to the tumour (figs 14 and 15). In this case secretory activity was marked and widespread in a tumour in which structural differentiation was minimal.

\section{Discussion}

We believe that the antibodies we used were active against milk-specific proteins, the principal of which was casein. There are several reasons for this belief: the antigen was prepared from human milk by a method for the isolation of casein; a single precipi-

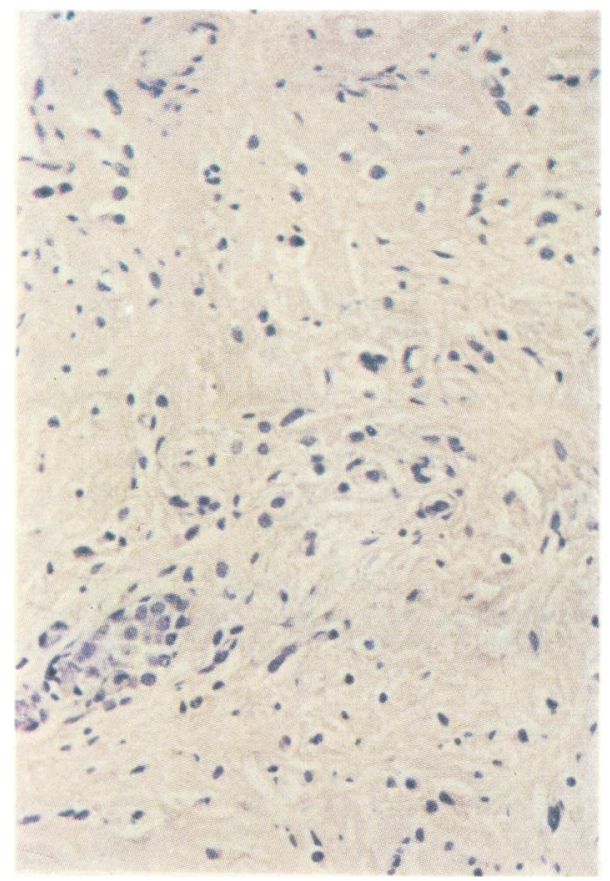

Fig 10

Fig 10 Poorly differentiated carcinoma (TRC 3679). Haematoxylin and eosin. $\times 200$.

Fig 11 Higher power view of part of fig 10 and same field as figure 12. The small amount of eosinophilic cytoplasm fluoresced brightly when previously stained by the indirect fluorescent antibody method. Section now overstained with haematoxylin and eosin. $\times 720$. tation line was seen in gel-diffusion plates between casein and antibody; the antibody reacted with human milk in histological sections but appropriate controls were unstained; and the ability of our antibodies to react was abolished by treatment with milk or casein but was unaffected by kidney, liver, or serum.

It is fortunate that fixation and histological processing do not destroy the antigenicity of casein for this allows the method to be used on paraffin sections (Young and Nelstrop, 1970). These are preferable to frozen sections and their use permits retrospective examination of cases for which important clinical data, such as response to hormone treatment and survival time, are known.

The three different distributions of secretory fluorescence that we have seen are interesting and require further investigation. The intraluminal fluorescence is probably due to protein that has already left the cells. It is not clear, however, whether it has followed either the focal or the diffuse form of distribution. The focal appearance may be a development by coalescence from the diffuse form or it may

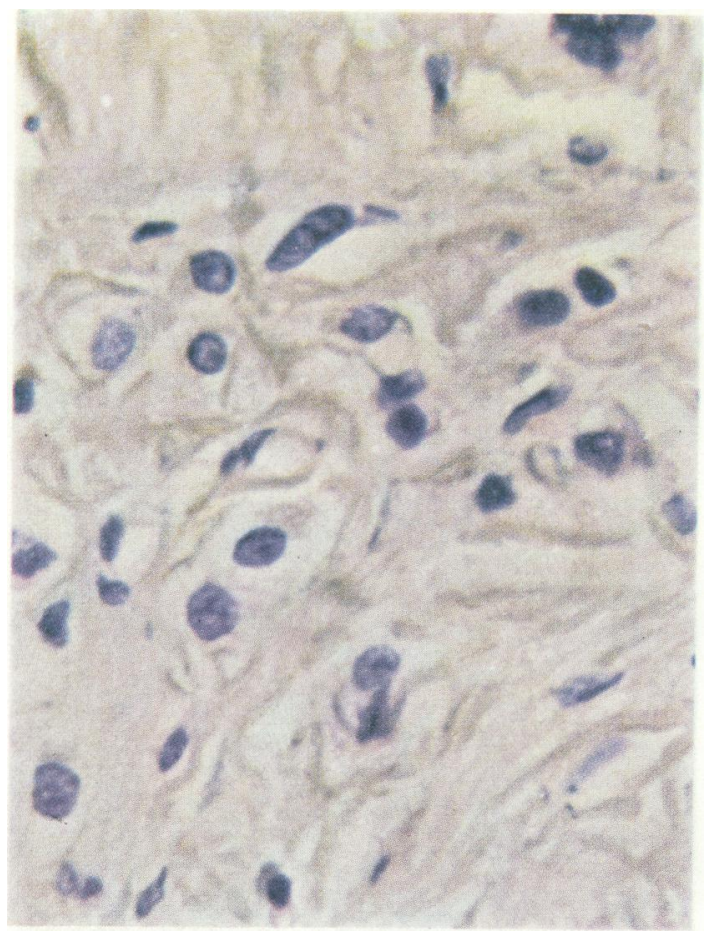

Fig 11 


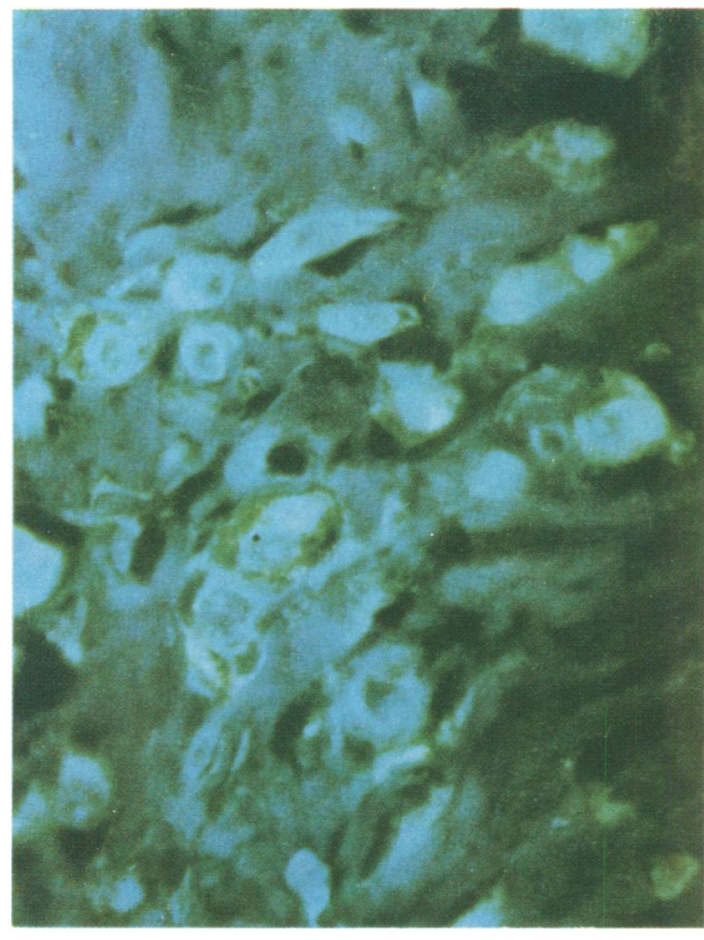

Fig 12 Same field as fig 11 with original staining by indirect fluorescent antibody method. Diffuse cytoplasmic fluorescence. $\times 720$.

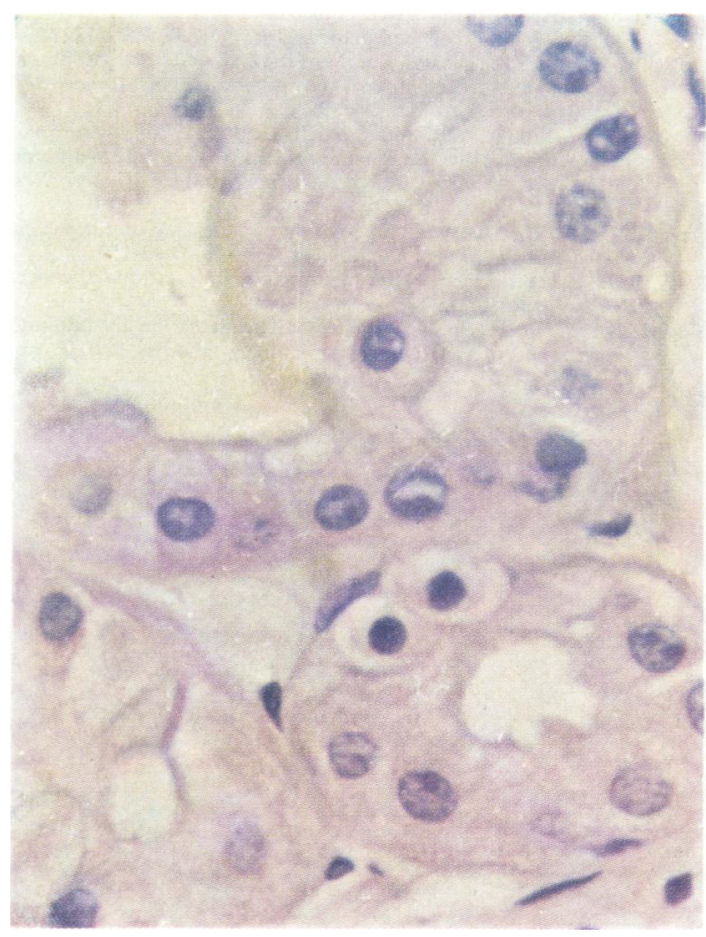

Fig 14 Duct lined by eosinophilic material that fluoresced brightly when previously stained by indirect fluorescent antibody method. Haematoxylin and eosin. $\times 720$.

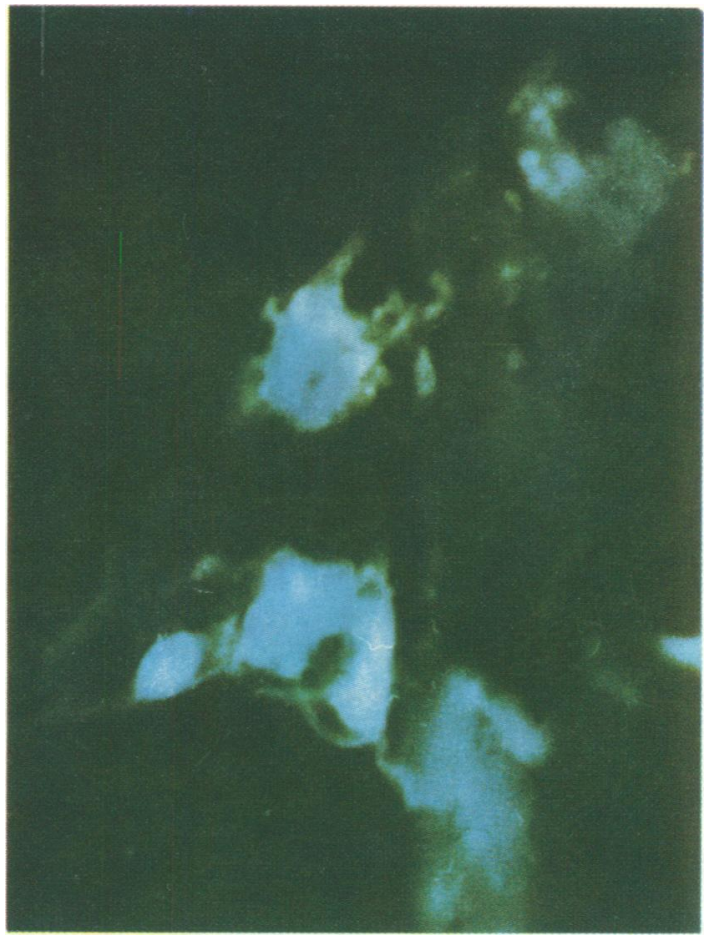

Fig 13 Higher power view of neoplastic cells from same tumour as those of figs 10,11, and 12. Indirect fluorescent antibody method. $\times 1800$.

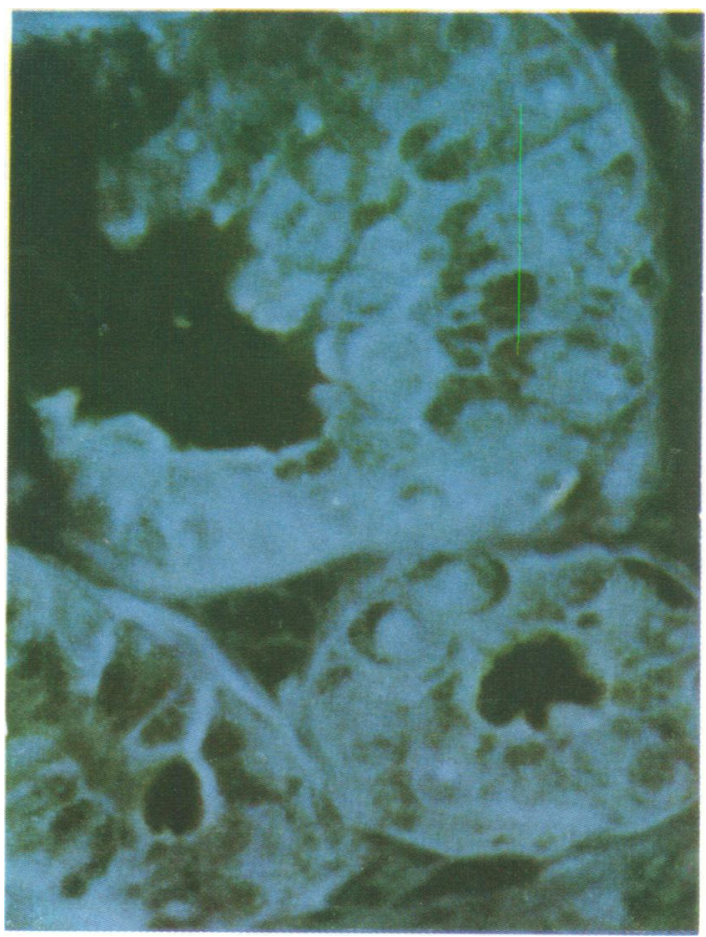

Fig 15 Same field as fig 14 with original staining by indirect fluorescent antibody method. Bright fluorescence on luminal border of duct. $\times 720$. 
be due to secretion into the intracellular lumina that have been seen in human breast cancer using the electron microscope (Ozzello, 1972).

Our results indicate that most breast cancers contain cells that secrete protein, most or all of which is probably casein. Our hypothesis is that caseinsecreting cells are functionally well differentiated and should respond to hormone interference by ceasing to proliferate. Tumours in which such casein is found would therefore be hormone-sensitive but before this hypothesis can be tested many more cases must be examined.

It is already clear from our results, however, that structural and functional differentiation in breast cancer are not always directly related to each other and the former is not necessarily a good guide to the latter. This could be an explanation of why some cancers with good structural differentiation are not hormone-sensitive in contrast to some apparently anaplastic cancers which are.

\section{Summary}

Paraffin sections from a series of 25 cases of human breast cancer were stained by the indirect fluorescent antibody technique using antibodies to milk-specific proteins (mainly casein).

All the cases showed some evidence of protein secretion either within the tumour or in the normal tissue adjacent to it. Secretion within the tumour was both extra- and intracellular. Extrace!lular secretion lined the luminal surface of neoplastic acini, intracellular secretion was focal or diffuse.

The secretion was not confined to areas of good structural differentiation but was also found in the cytoplasm of many invading cells that by histological standards would be called undifferentiated. It was not uniformly distributed but varied in amount from area to area of the tumour as well as from case to case.
We are indebted to Miss M. A. M. Bigbie of the Department of Obstetrics and Gynaecology and Miss E. Brinnand of the Department of Obstetrics, Central Middlesex Hospital, and to Miss $\mathbf{M}$. Babbege of the Department of Obstetrics, St Thomas's Hospital, for supplies of human milk; to Dr Rowland John, Department of Histopathology, St Thomas's Hospital, for sections of human lastating mammary gland; to Mr J. L. Hayward, Breast Unit, Guy's Hospital, and Dr G. A. K. Missen, Department of Pathology, Guy's Hospital, for histological sections of breast cancer; to Messrs Gerald and Austin Leach of the Department of Photography, Imperial Cancer Research Fund, for photographic assistance; to Messrs John Gilbert, Philip Attridge, and Kenneth Miller for technical assistance; and to Miss Marjorie Waight for typing the manuscript.

\section{References}

Avrameas, S., and Ternynck, T. (1967). Biologically active water insoluble protein polymers. Their use for isolation of antigen and antibodies. J. biol. Chem., 242, 1651-1659.

Beatson, G. T. (1896). On the treatment of inoperable cases of carcinoma of the mamma: suggestions for a new method of treatment with illustrative cases. Lancet, 2, 104-107 and 162165.

Bloom, H. J. G. (1950). Prognosis in carcinoma of the breast. Brit. J. Cancer, 4, 259-288.

Bloom, H. J. G., Richardson, W. W., and Harries, E. J. (1962). Natural history of untreated breast cancer (1805-1933). Comparison of untreated and treated cases according to histological grade of malignancy. Brit. med. J., 2, 213-221.

Huggins, C., and Bergenstal, D. M. (1952). Inhibition of human mammary and prostatic cancers by adrenalectomy. Cancer Res., 12, 134-141.

Knox, W. E., Linder, M., and Friedell, G. H. (1970). A series of transplantable rat mammary tumors with graded differentiation, growth rate, and glutaminase content. Cancer Res., 30, 283-287.

Ozzello, L. (1972). Ultrastructure of human mammary carcinoma cell in vivo and in vitro. J. nat. Cancer Inst., 48, 1043-150.

Ross, E. J. (1972). Endocrine and metabolic manifestations of cancer. Brit. med. J., 1, 735-738.

Young, S., and Nelstrop, A. E. (1970). The detection by immunofluorescence of casein in rat mammary glands. Brit. J. exp. Path., 51, 28-33. 